\title{
Qualidade de vida e saúde: um debate necessário
}

\author{
Quality of life and health: a necessary debate
}

Maria Cecília de Souza Minayo 1

Zulmira Maria de Araújo Hartz 2

Paulo Marchiori Buss 3

1 Vice-presidente de Ambiente, Comunicação e Informação, Fundação Oswaldo Cruz. Av. Brasil, 2.365, 21045-900, Rio de Janeiro, RJ, Brasil. mcmina@netra.castelo. fiocruz.br

2 Departamento de Epidemiologia e Métodos Quantitativos em Saúde,

Escola Nacional de Saúde Pública, Fundação

Oswaldo Cruz

3 Direção Escola Nacional de Saúde, Fundação

Oswaldo Cruz
Abstract This paper discusses the relationships between quality of life and health by applying the discourses emerging in the health sector to other fields and other disciplines. These relationships constitute social representation based on subjective parameters (well-being, happiness, love, pleasure, personal satisfaction), and on objective ones such as satisfaction of basic needs and of the needs created by the degree of economical and social development of a given society. The text presents the main instruments which have been constructed during the last years for measuring quality of life, as well as the debate they cause. It also debates the semantic field where the representations and actions in favour of quality of life - such as the concept of development, democracy, quality, way and conditions of life-develop. In relation to the field of health, this article discusses the tendency to restrict the concept of quality of life to the biomedical area, associated with an economic assessment. It shows the variety of instruments created for measuring quality of life in accordance with the concept. Health promotion is considered one of the most relevant strategies in this field in order to avoid medical reductionism and to develop an interdisciplinary dialogue. It is argued that this proposal, however, still needs to be refined and tested in sanitary practices.

Key words Quality of Life; Indicators for Quality of Life; Health Promotion
Resumo Este trabalho traz para o debate as relações entre saúde e qualidade de vida. Busca situar os discursos que se constróem na área da saúde em outros setores e outras disciplinas. Trata de uma representação social criada a partir de parâmetros subjetivos (bem-estar, felicidade, amor, prazer, realização pessoal), e também objetivos, cujas referências são a satisfação das necessidades básicas e das necessidades criadas pelo grau de desenvolvimento econômico e social de determinada sociedade. Mostra os principais instrumentos construídos nos últimos anos para medir qualidade de vida e as discussões que provocam. Reflete, também, sobre o campo semântico em que se desenvolvem as representações e ações voltadas para a qualidade de vida, como as noções de desenvolvimento, democracia, modo, condições e estilo de vida. Na área da saúde, discute a tendência de se estreitar o conceito de qualidade de vida ao campo biomédico, vinculando-o à avaliação econômica. Apresenta os mais variados instrumentos criados para medi-la nessa referida concepção. Considera a proposta de promoção da saúde como a mais relevante estratégia do setor, para evitar o reducionismo médico e realizar um diálogo intersetorial. Argumenta, porém, que essa proposta ainda carece de aprofundamento e de ser testada nas práticas sanitárias. Palavras-chave Qualidade de Vida; Indicadores de Qualidade de Vida; Promoção da Saúde 


\section{Introdução}

Tornou-se lugar-comum, no âmbito do setor saúde, repetir, com algumas variantes, a seguinte frase: saúde não é doença, saúde é qualidade de vida. Por mais correta que esteja, tal afirmativa costuma ser vazia de significado e, freqüentemente, revela a dificuldade que temos, como profissionais da área, de encontrar algum sentido teórico e epistemológico fora do marco referencial do sistema médico que, sem dúvida, domina a reflexão e a prática do campo da saúde pública. Dizer, portanto, que o conceito de saúde tem relações ou deve estar mais próximo da noção de qualidade de vida, que saúde não é mera ausência de doença, já é um bom começo, porque manifesta o malestar com o reducionismo biomédico. Porém, pouco acrescenta à reflexão.

Para realizar este trabalho, pesquisamos detalhadamente os anais dos congressos gerais e temáticos da Abrasco, assim como os registros de diversos seminários realizados pela instituição. Trata-se de material de grande relevância, já que saúde coletiva tem, nesses eventos científicos, sua expressão privilegiada. O termo qualidade de vida aparece sempre com sentido bastante genérico. Ora é empregado como título de seminários, chegando a designar o 2o Congresso de Epidemiologia, Qualidade de vida: compromisso histórico da epidemiologia (Lima e Costa \& Sousa, 1994), ora está associado a algumas classificações nos agrupamentos dos trabalhos dos vários congressos. Porém, em nenhum momento, existe uma definição dessa relação, seja no nível mais elementar de noção, e muito menos, como conceito. Isso quer dizer que se a idéia geral de qualidade de vida está presente, precisa ser mais bem explicitada e clarificada. $\mathrm{Na}$ abertura do 2o Congresso de Epidemiologia, Rufino Netto (1994) assim se refere: Vou considerar como qualidade de vida boa ou excelente aquela que ofereça um minimo de condições para que os indivíduos nela inseridos possam desenvolver o máximo de suas potencialidades, sejam estas: viver, sentir ou amar, trabalhar, produzindo bens e serviços, fazendo ciência ou artes. Falta o esforço de fazer da noção um conceito e tornálo operativo.

A área médica, por sua vez, já incorporou o tema qualidade de vida na sua prática profissional. Quando se apropria do termo, porém, o utiliza dentro do referencial da clínica, para designar o movimento em que, a partir de situações de lesões físicas ou biológicas, se oferecem indicações técnicas de melhorias nas condições de vida dos enfermos. A expressão usada é qualidade de vida em saúde. No entanto, a noção de saúde é totalmente funcional e corresponde ao seu contrário: a doença em causa, evidenciando uma visão medicalizada do tema. Os indicadores criados para medir esta qualidade de vida são notadamente bioestatísticos, psicométricos e econômicos, fundamentados em uma lógica de custo-benefício. $\mathrm{E}$ as técnicas criadas para medi-la não levam em conta o contexto cultural, social, de história de vida e do percurso dos indivíduos cuja qualidade de vida pretendem medir (Hubert, 1997).

Neste artigo, aceitamos o desafio de aprofundar a discussão sobre as relações entre saúde e qualidade de vida, por meio de um revisão sumária da literatura, buscando as bases conceituais e os fundamentos teórico-práticos de suas principais medidas. Trabalhamos a elaboração dos discursos e das técnicas de mensuração. Assim, esperamos poder contribuir para estabelecer um profícuo diálogo interdisciplinar, permitindo avançar no conhecimento e dar consistência a um tema que consideramos de grande importância tanto para a teoria como para a prática da saúde coletiva.

\section{Qualidade de vida: uma noção polissêmica}

Quanto mais aprimorada a democracia, mais ampla é a noção de qualidade de vida, o grau de bem-estar da sociedade e de igual acesso a bens materiais e culturais (Olga Matos, 1999).

Qualidade de vida é uma noção eminentemente humana, que tem sido aproximada ao grau de satisfação encontrado na vida familiar, amorosa, social e ambiental e à própria estética existencial. Pressupõe a capacidade de efetuar uma síntese cultural de todos os elementos que determinada sociedade considera seu padrão de conforto e bem-estar. O termo abrange muitos significados, que refletem conhecimentos, experiências e valores de indivíduos e coletividades que a ele se reportam em variadas épocas, espaços e histórias diferentes, sendo portanto uma construção social com a marca da relatividade cultural. Auquier et al. (1997) a qualificam como um conceito equívoco como o de inteligência, ambos dotados de um senso comum variável de um indi- 
víduo ao outro. (Martin \& Stockler, 1998) sugerem que qualidade de vida seja definida em termos da distância entre expectativas individuais e a realidade (sendo que quanto menor a distância, melhor).

A relatividade da noção, que em última instância remete ao plano individual, tem pelo menos três fóruns de referência. O primeiro é histórico. Ou seja, em determinado tempo de seu desenvolvimento econômico, social e tecnológico, uma sociedade específica tem um parâmetro de qualidade de vida diferente da mesma sociedade em outra etapa histórica. O segundo é cultural. Certamente, valores e necessidades são construídos e hierarquizados diferentemente pelos povos, revelando suas tradições. O terceiro aspecto se refere às estratificações ou classes sociais. Os estudiosos que analisam as sociedades em que as desigualdades e heterogeneidades são muito fortes mostram que os padrões e as concepções de bemestar são também estratificados: a idéia de qualidade de vida está relacionada ao bem-estar das camadas superiores e à passagem de um limiar a outro.

O relativismo cultural, no entanto, não nos impede de perceber que um modelo hegemônico está a um passo de adquirir significado planetário. É o preconizado pelo mundo ocidental, urbanizado, rico, polarizado por um certo número de valores, que poderiam ser assim resumidos: conforto, prazer, boa mesa, moda, utilidades domésticas, viagens, carro, televisão, telefone, computador, uso de tecnologias que diminuem o trabalho manual, consumo de arte e cultura, entre outras comodidades e riquezas.

A partir do crescimento do movimento ambientalista na década de 1970, o questionamento dos modelos de bem-estar predatórios, agregaram, à noção de conforto, bem-estar e qualidade de vida, a perspectiva da ecologia humana - que trata do ambiente biogeoquímico, no qual vivem o indivíduo e a população; e o conjunto das relações que os seres humanos estabelecem entre si e com a própria natureza. Esse conceito não aplica a dimensão evolucionista de uma escalada cada vez maior de conforto, consumo e bem-estar. Pelo contrário, ele se apóia na idéia de excelência das condições de vida (Witier, 1997) e de desenvolvimento sustentável. Questiona as condições reais e universais de manutenção de um padrão de qualidade de vida fundado no consumismo e na exploração da natureza que, pelo seu elevado grau predatório, desdenha a situação das gerações futuras, desconhece a cumplicidade de toda a biosfera e não é replicável.

No campo da saúde, o discurso da relação entre saúde e qualidade de vida, embora bastante inespecífico e generalizante, existe desde o nascimento da medicina social, nos séculos XVIII e XIX, quando investigações sistemáticas começaram a referendar esta tese e dar subsídios para políticas públicas e movimentos sociais. A situação da classe trabalhadora na Inglaterra, de Engels, ou Mortalidade diferencial na França, de Villermé, ambas citadas por Rosen (1980), são exemplos de tal preocupação. Na verdade, a idéia dessa relação atravessa toda a história da medicina social ocidental e também latino-americana, como mostram os trabalhos de Mckeown (1982), Breilh et al. (1990), Nuñez (1994) e Paim (1994). De fato, na maioria dos estudos, o termo de referência não é qualidade de vida, mas condições de vida. Como mencionado em Witier (1997), estilo de vida e situação de vida são termos que compõem parte do campo semântico em que o tema é debatido.

A visão da intrínseca relação entre condições e qualidade de vida e saúde aproxima os clássicos da medicina social da discussão que, nos últimos anos, vem se revigorando na área, e tem no conceito de promoção da saúde sua estratégia central. Redimensionado pelo pensamento sanitarista canadense a partir do conhecido relatório Lalonde (1974), tal conceito foi definido, tomando como base na concepção atual do que se consideram os determinantes da saúde: 1) o estilo de vida; 2) os avanços da biologia humana; 3 ) o ambiente físico e social e 4) serviços de saúde. Conferências mundiais e regionais (MS, 1997) têm debatido e ampliado o sentido do conceito de promoção que, a nosso ver, constitui a estratégia chave da discussão da qualidade de vida pelo setor (Buss et al., 1998). O tema da promoção é objeto específico de um artigo deste número da revista.

Por fim, é importante observar também que, em todas as sondagens feitas sobre qualidade de vida, valores não materiais, como amor, liberdade, solidariedade e inserção social, realização pessoal e felicidade, compõem sua concepção. Como lembra Witier (1997), para o ser humano, o apetite da vida está estreitamente ligado ao menu que lhe é oferecido. Seria, portanto, qualidade de vida uma mera representação social? Sim e não. Sim, pelos ele- 
mentos de subjetividade e de incorporação cultural que contém. Não, porque existem alguns parâmetros materiais na construção desta noção que a tornam também passível de apreciação universal, como veremos a seguir.

O patamar material mínimo e universal para se falar em qualidade de vida diz respeito à satisfação das necessidades mais elementares da vida humana: alimentação, acesso a água potável, habitação, trabalho, educação, saúde e lazer; elementos materiais que têm como referência noções relativas de conforto, bem-estar e realização individual e coletiva. No mundo ocidental atual, por exemplo, é possível dizer também que desemprego, exclusão social e violência são, de forma objetiva, reconhecidos como a negação da qualidade de vida. Trata-se, portanto, de componentes passíveis de mensuração e comparação, mesmo levando-se em conta a necessidade permanente de relativizá-los culturalmente no tempo e no espaço.

Em resumo, a noção de qualidade de vida transita em um campo semântico polissêmico: de um lado, está relacionada a modo, condições e estilos de vida (Castellanos, 1997). De outro, inclui as idéias de desenvolvimento sustentável e ecologia humana. E, por fim, relaciona-se ao campo da democracia, do desenvolvimento e dos direitos humanos e sociais. No que concerne à saúde, as noções se unem em uma resultante social da construção coletiva dos padrões de conforto e tolerância que determinada sociedade estabelece, como parâmetros, para si.

\section{Qualidade de vida: medidas e padrões gerais}

A medida de qualidade de vida, mesmo se é ainda um instrumento recente e vindo de uma tradição estrangeira, anglo-saxônica, empirista e utilitarista, é um fato irreversivel que vai, provavelmente, pertencer ao nosso universo, da mesma forma que a ecografia (Rameix, 1997:89).

Tentando sintetizar a complexidade da noção de qualidade de vida e de sua relatividade $v i s$-à-vis as diferentes culturas e realidades sociais, diversos instrumentos têm sido construídos. Alguns tratam a saúde como componente de um indicador composto, outros têm, no campo da saúde, seu objeto propriamente dito.

Entre os primeiros, talvez o mais conhecido e difundido seja o Índice de Desenvolvi- mento Humano (IDH), elaborado pelo Programa das Nações Unidas para o Desenvolvimento (PNUD). O IDH foi criado com a intenção de deslocar o debate sobre desenvolvimento de aspectos puramente econômicos como nível de renda, produto interno bruto e nível de emprego - para aspectos de natureza social e também cultural. Embutida nesse indicador encontra-se a concepção de que renda, saúde e educação são três elementos fundamentais da qualidade de vida de uma população.

O IDH é um indicador sintético de qualidade de vida que, de forma simplificada, soma e divide por três os níveis de renda, saúde e educação de determinada população. A renda é avaliada pelo PIB real per capita; a saúde, pela esperança de vida ao nascer e a educação, pela taxa de alfabetização de adultos e taxas de matrículas nos níveis primário, secundário e terciário combinados. Renda, educação e saúde seriam atributos com igual importância como expressão das capacidades humanas.

O IDH se baseia na noção de capacidades, isto é, tudo aquilo que uma pessoa está apta a realizar ou fazer. Nesse sentido, o desenvolvimento humano teria, como significado mais amplo, a expansão não apenas da riqueza, mas da potencialidade dos indivíduos de serem responsáveis por atividades e processos mais valiosos e valorizados. Assim, a saúde e a educação são estados ou habilidades que permitem uma expansão das capacidades. Inversamente, limitações na saúde e na educação seriam obstáculos à plena realização das potencialidades humanas (PNUD, 1990).

O IDH vem recebendo aceitação ampla pelas facilidades na obtenção dos índices que o compõem - disponíveis na maioria dos países e regiões do mundo e são construídos com metodologia semelhante -, o que garante razoável grau de aplicabilidade entre realidades totalmente diversas. Mas também apresenta limitações que devem ser consideradas, seja no uso para comparar qualidade de vida entre territórios, seja ao longo do tempo em um mesmo território. Por exemplo, discrimina pouco os países ou regiões mais desenvolvidas entre si, pois aí, as taxas de analfabetismo têm diferenças irrisórias, e apresenta problemas de consistência metodológica quando aplicado a limites geográficos mais restritos, nos quais provavelmente os rankings produzidos seriam meras reproduções, com poucas diferenças, da diferenciação da renda (Cardoso, 1998). 
Finalmente, o IDH não consegue incorporar a essência do conceito central que tenta medir. A esse respeito se refere Dines (1999): desenvolvimento é um processo mais amplo que o mero aumento da promoção, melhoria de produção e de índices. Envolve a direção, o sentido e sobretudo o conteúdo do crescimento. Atualmente, essa dimensão anímica do processo econômico faz a diferença entre o crescimento e o desenvolvimento. Um país pode crescer ou deixar de crescer. Mas uma nação desenvolvida nunca pode deixar de sê-lo, porque o desenvolvimento se incorpora às estruturas, às instituições e às mentalidades. E não se desencarna. Da mesma forma, o campo semântico da qualidade de vida na tradição ocidental, além da idéia de desenvolvimento, transita pela crença na democracia. Quanto mais aprimorada a democracia, mais ampla é a noção de qualidade de vida, do grau de bem-estar da sociedade e da eqüidade ao acesso aos bens materiais e culturais. Manifesta-se de forma palpável na dimensão de convivência entre as pessoas, reveladora de urbanidade e respeito mútuo. Nesse sentido, a força espiritual da democracia é um fator de resistência à redução de todas as esferas da vida, ao fato econômico (Matos, 1998).

Poderia ser criticado ainda do ponto de vista ético-filosófico, na medida que revelaria um viés etnocêntrico, que toma os padrões ocidentais modernos como modelos de referência a serem atingidos por todas as nações do planeta. Por exemplo, em países em desenvolvimento com baixo grau de institucionalização das relações mercantis, a renda é um critério pouco efetivo para avaliar a produção e a circulação de bens e riquezas. Por outro lado, existem sociedades em que o acesso ao conhecimento se dá a partir de meios ligados à tradição ou à transmissão oral, mais eficazes para lidar com as realidades locais do que a alfabetização (Cardoso, 1998).

Apesar das justas críticas que tem recebido, o IDH tem sido bastante utilizado, inclusive no Brasil, e inspirado outros como o Índice de Condições de Vida (ICV). Desenvolvido pela Fundação João Pinheiro, em Belo Horizonte, para estudar a situação de municípios mineiros, foi logo depois adequado, em consórcio com o IPEA, o IBGE e o PNUD, para a análise de todos os municípios brasileiros (IPEA/IBGE/FJP/PNUD, 1998). Instrumento muito mais sofisticado do que o IDH, com a vantagem de poder ser aplicado para micror- realidades, o ICV é um composto de 20 indicadores em cinco dimensões: 1 ) renda (familiar per capita, grau de desigualdade, percentagem de pessoas com renda insuficiente, insuficiência média de renda e grau de desigualdade na população de renda insuficiente); 2) educação (taxa de analfabetismo, número médio de anos de estudo, percentagem da população com menos de 4 anos de estudo, percentagem da população com menos de 8 anos de estudo e percentagem da população com mais de 11 anos de estudo); 3) infância (percentagem de crianças que trabalham, percentagem de crianças que não freqüentam escola, defasagem escolar média e percentagem de crianças com mais de um ano de defasagem escolar); 4) habitação (percentagem da população em domicílios com densidade média acima de duas pessoas por dormitório, percentagem da população que vive em domicílios duráveis e percentagem da população que vive em domicílios com instalações adequadas de esgoto) e 5) longevidade (esperança de vida ao nascer e taxa de mortalidade infantil). O ICV é sintetizado por meio de vários artifícios metodológicos, podendo ser compreendido em toda a sua extensão no trabalho 'Desenvolvimento humano e condições de vida' resultado da colaboração entre FJP/IPEA/IBGE/PNUD (1998).

Mesmo tendo seu espectro de abrangência muito mais ampliado, o ICV trabalha apenas com os aspectos objetivos, passíveis de medição. É fundamental sua contribuição? Sem dúvida, na medida que existe uma intrínseca relação entre a busca de eqüidade social e a capacidade de desenvolvimento intelectual, de aspirações e de reivindicação de determinada população ou grupos sociais.

Além deste conhecido indicador composto, identificam-se diversos outros, objetivos e subjetivos, que expressam alguma dimensão da qualidade de vida. Os considerados objetivos referem-se sempre a situações como renda, emprego/desemprego, população abaixo da linha da pobreza, consumo alimentar, domicílios com disponibilidade de água limpa, tratamento adequado de esgoto e lixo e disponibilidade de energia elétrica, propriedade da terra e de domicílios, acesso a transporte, qualidade do ar, concentração de moradores por domicílio e outras.

Os de natureza subjetiva respondem a como as pessoas sentem ou o que pensam das suas vidas, ou como percebem o valor dos componentes materiais reconhecidos como 
base social da qualidade de vida. Deste último caso pode ser exemplo, o Índice de Qualidade de Vida (IQV) de São Paulo, criado pelo jornal Folha de S. Paulo, que inclui um conjunto de nove fatores (trabalho, segurança, moradia, serviços de saúde, dinheiro, estudo, qualidade do ar, lazer e serviços de transporte). Esses elementos são analisados a partir do ponto de vista da população, que é dividida por faixa de renda, escolaridade, categoria social, sexo e faixa etária. A pergunta-chave é o grau de satisfação dos cidadãos, classificado em satisfatório, insatisfatório e péssimo, em um intervalo de 0 a 10 (Índice Folha, 1999). Os considerados objetivos referem-se a aspectos globais e gerais da vida, assim como a satisfação com domínios específicos da existência. Pesquisadores da Universidade de Michigan, por exemplo, citados por Patrick \& Erickson (1993), avaliaram a importância de cada domínio e de seus componentes específicos para a satisfação global com o domínio. Os níveis de bemestar e felicidade foram então correlacionados a características sociais, geográficas e demográficas específicas.

Parece-nos claro, ainda, que a qualidade de vida não é definível exclusivamente a partir de critérios científicos ou técnicos. Por essa razão, alguns autores remetem a discussão também para o âmbito político. Ou seja, os parâmetros para compor um padrão mínimo que permita a construção de agendas de intervenção ou a avaliação de políticas não são autoevidentes ou factíveis apenas em gabinetes e laboratórios, devendo resultar de debates sociais amplos, que estabeleçam consensos mínimos.

No Brasil, um exemplo significativo desse modelo é o IQV de Belo Horizonte, criado a partir de um levantamento das questões consideradas relevantes pela população e tendo como objetivo fundamentar os debates públicos sobre o orçamento participativo. No entanto, mais do que um índice sintético, o IQV/BH seria um indicador setorial de carências, permitindo não apenas hierarquizar áreas, mas também identificar problemas a serem enfrentados em cada bairro.

Pode-se observar, por fim, que nenhum componente propriamente médico (ou sequer de indicadores clássicos de morbi-mortalidade) entra na composição dos indicadores compostos de qualidade de vida. Ou seja, tanto o IDH, o ICV, como outros já citados tratam a saúde como um dos componentes de uma complexa resultante social. Concordando, em termos gerais com esse ponto de vista, nós neste trabalho a entendemos como uma síntese, um híbrido biológico-social, mediado por condições mentais, ambientais e culturais.

\section{Qualidade de vida: medidas padrões do setor saúde}

As definições ampliadas já descritas convivem com outras mais restritas e específicas, como as econômicas e como as que também têm sido desenvolvidas no setor saúde e que, como já dissemos, quase sempre se resumem ao campo médico. A expressão qualidade de vida ligada à saúde (QVLS) é definida por Auquier et al. (1997) como o valor atribuído à vida, ponderado pelas deteriorações funcionais; as percepções e condições sociais que são induzidas pela doença, agravos, tratamentos; e a organização política e econômica do sistema assistencial. A versão inglesa do conceito de healthrelated quality of life (HRQL), em Gianchello (1996), é similar: é o valor atribuído à duração da vida quando modificada pela percepção de limitações físicas, psicológicas, funções sociais e oportunidades influenciadas pela doença, tratamento e outros agravos, tornando-se o principal indicador para a pesquisa avaliativa sobre o resultado de intervenções. Sendo utilizado nessa conotação, o HRQL indicará também se o estado de saúde medido ou estimado é relativamente desejável (Gold et al., 1996). Para esses autores, os conceitos fundamentais de HRQL seriam igualmente a percepção da saúde, as funções sociais, psicológicas e físicas, bem como os danos a elas relacionados.

Mostrando a extrema variabilidade do conceito, a revisão de Ann Bowling (1991) sobre as escalas de qualidade de vida relacionadas com saúde inclui medidas de capacidade funcional, do estado de saúde, de bem-estar psicológico, de redes de apoio social, de satisfação e estado de ânimo de pacientes. Em geral, de forma implícita ou explícita, toda medida repousa sobre teorias que guiam a seleção de procedimentos de mensuração. Auquier et al. (1997) consideram que três correntes orientam a construção dos instrumentos hoje disponíveis: o funcionalismo, que define um estado normal para certa idade e função social e seu desvio, ou morbidade, caracterizado por indicadores individuais de capacidade de exe- 
cução de atividades; a teoria do bem-estar, que explora as reações subjetivas das experiências de vida, buscando a competência do indivíduo para minimizar sofrimentos e aumentar a satisfação pessoal e de seu entorno e a teoria da utilidade, de base econômica, que pressupõe a escolha dos indivíduos ao compararem um determinado estado de saúde a outro.

Em relação ao campo de aplicação, as medidas podem ser classificadas como genéricas, se usam questionários de base populacional sem especificar patologias, sendo mais apropriadas a estudos epidemiológicos, planejamento e avaliação do sistema de saúde. Um desses instrumentos foi desenvolvido pela OMS que recentemente criou o Grupo de Qualidade de Vida, The WHOQOL Group (1995), e definiu o termo como a percepção do indivíduo de sua posição na vida, no contexto da cultura e do sistema de valores em que vive e em relação aos seus objetivos, expectativas, padrões e preocupações. Assim, o instrumento desenvolvido por esse organismo internacional em estudo multicêntrico baseia-se nos pressupostos de que qualidade de vida é uma construção subjetiva (percepção do indivíduo em questão), multidimensional e composta por elementos positivos (por exemplo, mobilidade) e negativos (dor).

O grupo desenvolveu, até o momento, dois instrumentos gerais de medida de qualidade de vida: o WHOQOL-100 e o WHOQOL-Bref. O primeiro consta de 100 questões que avaliam seis domínios: a) físico, b) psicológico, c) de independência, d) relações sociais, e) meio ambiente e f) espiritualidade/crenças pessoais. $\mathrm{O}$ segundo instrumento é uma versão abreviada, com 26 questões, extraídas do anterior, entre as que obtiveram os melhores desempenhos psicométricos, cobrindo quatro domínios: a) físico, b) psicológico, c) relações sociais e d) meio ambiente. A versão em português inclusive dos questionários - está disponível no Brasil, no Grupo de Estudos sobre Qualidade de Vida, do Departamento de Psiquiatria da Universidade Federal do Rio Grande do Sul e no Hospital das Clínicas do Paraná.

Outras modalidades de mensuração podem ser classificadas como específicas. Muitas podem ser encontradas na literatura sobre saúde e qualidade de vida, mormente nas fontes anglo-saxãs (volumes e fascículos do periódico Quality of Life Research). Os estudos apontam, em geral, para situações relacionadas à qualidade da vida cotidiana dos indivíduos, subseqüente à experiência de doenças, agravos ou intervenções médicas. Referem-se a doenças crônicas - como câncer, diabete, doença coronariana e cerebrovascular, Parkinson e outros problemas do sistema nervoso, hepatites e artrites crônicas, asma brônquica e outras doenças respiratórias - ou a conseqüências crônicas (seqüelas ou medidas curativas e reabilitadoras) de doenças ou agravos agudos, como problemas neurológicos póstraumáticos, transplantes, uso de insulina e outros medicamentos de uso prolongado. Vários instrumentos incluem indicadores para aspectos subjetivos da convivência com doenças e lesões, como sentimentos de vergonha e culpa, que trazem conseqüências negativas sobre a percepção da qualidade de vida por parte dos indivíduos acometidos e suas famílias.

Bley et al. (1997) falam da multiplicidade de usos profanos do conceito, particularmente no domínio da comunicação e do consumo e alertam para o fato de que, embora a OMS considere que se deveria prioritariamente medir a qualidade de vida de cinco grupos (pacientes crônicos, seus familiares e pessoal de suporte, pessoas em situações extremas, com dificuldade de comunicação, e crianças), os estudo têm-se concentrado nos pacientes crônicos, o que é facilmente evidenciável nas bases bibliográficas. Hubert (1997) julga também que a literatura sobre qualidade de vida é essencialmente medicalizada, adotando uma visão bioestatística e economicista da saúde. Durand et al. (1997) acrescentam que os estudos são funcionalistas e focalizados no custoefetividade.

No âmbito médico, desenvolveram-se também instrumentos de avaliação de qualidade de vida, focalizados, primeiramente, sobre a idéia de complementar as análises de sobrevida. Esses estudos evoluíram para integrar análises de custo-utilidade, em voga na década de 1980, que ampliavam a visão restrita nos trabalhos de custo-eficácia dos anos 70, criticados por se deterem apenas em indicadores clínicos (Hartz \& Pouvourville, 1998). A argumentação tornou-se, assim, a de que a qualidade de vida dos pacientes deveria alcançar um patamar melhor do que o anterior à intervenção (Auray \& Duru, 1995). Desde então, passou-se a considerar que os estudos de custo-utilidade são apropriados quando a qualidade de vida é um resultado importante, usualmente apresentado como custo por ano 
de vida ganho, ajustado pela qualidade ou QALY (quality-adjusted life-years).

Auquier et al. (1997) propõem uma taxonomia das medidas, que vão de indicadores simples a baterias ou conjunto de instrumentos, com indicadores e metrologias específicas. Mesmo que não permitam agregar dados, segundo os autores, sua importância residiria na possibilidade de comparação de resultados. Nesse sentido, o QALY seria o mais apropriado, por combinar abordagem de quantidade e qualidade de vida em uma estimativa de custo-oportunidade, para orientar a decisão de alocação de recursos, envolvendo profissionais de saúde pública e economistas. Porém, sobre a interpretação multiprofissional, Lebrun \& Sailly (1996) destacam contradições, uma vez que os problemas suscitados para os economistas, na definição e medida de qualidade de vida, são diferentes dos que têm médicos e psicometristas pois, para a medicina baseada em evidências, o único critério é a eficácia clínica.

Matematicamente, o QALY é calculado como a soma do produto de anos de vida e a qualidade de vida em cada um desses anos. A um ano de vida em ótima saúde é atribuído o valor 1 (um) e o valor 0 (zero) para o óbito (Dasbach \& Teutsch, 1996). O estado de saúde pode ser medido direta ou indiretamente. Na forma direta, é o indivíduo que valoriza seu estado de saúde feito sob a forma de loteria, indagando-se sobre a escolha de um estado desejável, a probabilidade de melhorá-lo e a morte. A abordagem indireta refere-se a preferências do público em geral. A qualidade de vida é estimada usando dados que combinam diversas dimensões para computar uma série de valores atribuídos matematicamente no modelo multi-attribute-utility (MAU).

As incertezas do QALY são relacionadas por Briggs (1995): a) tipos de dados requeridos - indicadores de recursos e estados conseqüentes a intervenções ou tecnologias comparadas só seriam otimizados com o uso simultâneo de avaliação clínica e econômica na mesma população; b) extrapolação de dados, referindo-se a resultados clínicos intermediários, extrapolados para finalísticos; c) generalização de resultados evidenciando dificuldades relacionadas a diferenças demográficas, epidemiológicas, preços e custos e variações na prática clínica e d) discordância na escolha de métodos analíticos e de metodologias entre economistas, assim como problemas ético-morais referentes a escolhas.
Para Maynard \& Bloom (1998), o idadeísmo é um dos principais problemas do QALY, na medida que embute preconceito contra o idoso, considerando-o sempre com menor expectativa e menor qualidade de sobrevida, no que se refere a procedimentos médicos. Observa-se a mesma lógica discriminatória em relação aos portadores de deficiência física, pois jamais partem de uma qualidade de vida $=1$. Mesmo no sistema de saúde inglês, onde o QALY se originou, tentou-se generalizar seu uso a qualquer novo tratamento com financiamento público, mas somente em 27 dos 95 projetos foi possível calcular e em apenas 10 se pôde comparar com o tratamento anterior (Castiel, 1995). Segundo Castiel, embora um fator como o QALY ajude a decisão sobre o que fazer, não pode seguir apenas a lógica matemática. Assim, muitos estudos se tornam inúteis e alguns chegam a considerar o uso do QALY efeito perverso ampliado da esperança de vida ao nascer (Castiel, 1995).

Para Green (1995), a questão ética chave é: quem fará as escolhas subjetivas que determinam o QALY? Seriam os profissionais de saúde, o público em geral ou os pacientes que vivenciam as condições analisadas, uma vez que são três lógicas diferentes em jogo? Por esse motivo, Schlenk et al. (1998) demonstram que é preciso coletar opiniões diversas ao comparar portadores de doenças crônicas com pessoas saudáveis. Oleske (1995) comenta que um dos problemas, dentro da perspectiva epidemiológica, é que as medidas não contemplam qualquer pressuposto sobre a intensidade ou duração dos sintomas ou acerca da existência de patologias associadas.

Outros autores, como Moatti (1996), advertem para os perigos do utilitarismo, que apresenta pelo menos dois problemas. Primeiro, a lei de rendimentos decrescentes do sistema de saúde, pois quanto mais se ampliam as indicações de tecnologias, maiores são os custos por unidade de resultado. Outro perigo seria a tentação, para a saúde pública, de querer hierarquizar os custos por QALY.

Uma variante do QALY apareceu em 1994, quando a OMS publicou uma série de trabalhos, cujo objetivo era a medida da carga global de doenças (global burden disease, GBD) em diversas regiões do mundo, como descritora do estado de saúde das populações. Esperava-se que o novo indicador fosse capaz de superar as insuficiências do QALY, na medida que: a) incorpora condições não fatais na apre- 
ciação do status da saúde; b) desliga epidemiologia de advocacia, visando a produzir avaliação objetiva e demograficamente plausível da carga de doenças e outras condições particulares e c) mede a carga de doença e incapacidade em termos de custo por unidade de caso evitado, valorizando o custo-efetividade das intervenções.

O QALY posteriormente foi substituído por DALY (disability-adjusted life-years), em português, anos de vida corrigidos pela incapacidade (AVCI). A mudança fundamental entre um e outro é que o DALY, em lugar de buscar o valor subjetivo atribuído pelos indivíduos a cada um dos estados de saúde, é construído a partir da mortalidade estimada para cada doença e seu efeito incapacitante, ajustado pela idade das vítimas; e uma taxa de atualização, para calcular o valor de uma perda futura. O conceito de incapacidade foi definido com a arbitragem exclusiva de especialistas internacionais, segundo eles, buscando o máximo de objetividade (Brunet-Jailly, 1997). Para calcular o DALY total de uma determinada condição (acidentes de trânsito em uma auto-estrada, por exemplo), soma-se o número de anos perdidos em óbitos prematuros por essa causa e o total de anos vividos com incapacidades de conhecida severidade e duração, pelos sobreviventes de tais acidentes. Um óbito prematuro é definido como aquele que ocorre antes da idade que se esperaria sobreviver ao se padronizar a expectativa de vida pela mais longa do mundo, no caso a do Japão, hoje em 82,5 anos para a mulher e 80 anos para o homem.

Para se calcular o número de anos vividos com uma condição incapacitante, parte-se da incidência, a idade média de início da doença, a média de duração da incapacidade (meses ou anos) e a severidade dos casos com ou sem tratamento. O cálculo de severidade se baseia em um conjunto de indicadores de 22 condições classificadas em 7 níveis, ponderadas de 0 (zero) a 1 (um).

Apesar das dificuldades de se ponderar os referidos valores, os autores relatam uma grande concordância intercultural de profissionais de vários países do mundo, na arbitragem ou nas negociações para consenso de graus de severidade e outras implicações das escolhas de quantidade e qualidade de vida. Essas opções afrontam o que seria moralmente aceitável, porém são praticadas implicitamente nos sistemas de atenção à saúde em todo mundo. $\mathrm{O}$
DALY foi utilizado no Relatório Anual do Banco Mundial de 1993, comparando a carga de doenças nas diversas regiões do mundo e o custo-efetividade de uma variedade de intervenções que lidam com esses problemas (Hinman, 1997). O propósito é redirecionar os recursos das intervenções ditas de maior custo por DALY ganho, de modo a garantir um pacote mínimo que reduza a carga das doenças, sem aumentar os recursos da saúde. Para Dasbach \& Teutsch (1996), embora o Banco Mundial venha usando o DALY para comparar condições de saúde, falta-lhe o rigor de outras medidas de preferência, uma vez que esse indicador utiliza apenas as preferências de um grupo de especialistas.

$\mathrm{O}$ indicador healthy life-year (HeaLY), que combina anos de vida perdidos pela morbidade com os que são atribuídos a mortalidade prematura e pode ser aplicado a indivíduos e a populações, foi comparado com o DALY por Hyder et al. (1998), demonstrando ser mais compreensível, mais simples e flexível. Esses atributos, que facilitam sua utilização para tomada de decisão, lhes pareceram suficientes para que o recomendassem como medida da carga de doença ou identificar grupos mais vulneráveis, ao se avaliarem o custo e os benefícios dos programas de intervenção.

\section{Potencial das medidas de qualidade de vida e a busca da promoção da saúde: orientando o debate}

Como se pode concluir, o tema qualidade de vida é tratado sob os mais diferentes olhares, seja da ciência, através de várias disciplinas, seja do senso comum, seja do ponto de vista objetivo ou subjetivo, seja em abordagens individuais ou coletivas. No âmbito da saúde, quando visto no sentido ampliado, ele se apóia na compreensão das necessidades humanas fundamentais, materiais e espirituais e tem no conceito de promoção da saúde seu foco mais relevante. Quando vista de forma mais focalizada, qualidade de vida em saúde coloca sua centralidade na capacidade de viver sem doenças ou de superar as dificuldades dos estados ou condições de morbidade. Isso porque, em geral, os profissionais atuam no âmbito em que podem influenciar diretamente, isto é, aliviando a dor, o mal-estar e as doenças, intervindo sobre os agravos que podem gerar dependências e desconfortos, seja para evitá-los, 
seja minorando conseqüências dos mesmos ou das intervenções realizadas para diagnosticá-los ou tratá-los.

Assim, ainda que reconheça que poderosos determinantes estejam freqüentemente situados fora do setor e bastante ligados ao que se consideraria, no senso comum, como componentes da qualidade de vida, o sistema de saúde não intervém sobre eles; sente-se impotente ou simplesmente passa ao largo de tais relações. Na maioria das vezes, adota uma posição exclusivamente retórica quanto aos chamados determinantes extra-setoriais que são, em grande parte, os mais relevantes componentes da qualidade de vida e também de uma vida saudável. Até mesmo o papel de mediação intersetorial e entre a população sob risco ou em situação de vulnerabilidade e o poder público - bastante preconizado como estratégia para a promoção da saúde - tem sido pouco acionado pelo setor, na maior parte dos países do mundo.

Por outro lado, é preciso assinalar também que, embora se saiba que o estado de saúde de indivíduos e coletividades, assim como o sistema de saúde, influenciam e são influenciados pelo ambiente global, há que se reconhecer que nem todos os aspectos da vida humana são, necessariamente, uma questão médica ou sanitária. A ação governamental ou comunitária sobre os mesmos está compartimentalizada em setores econômicos e sociais e distribuída entre diferentes grupos de interesse e organizações. Desse modo, pode-se dizer que a questão da qualidade de vida diz respeito ao padrão que a própria sociedade define e se mobiliza para conquistar, consciente ou inconscientemente, e ao conjunto das políticas públicas e sociais que induzem e norteiam o desenvolvimento humano, as mudanças positivas no modo, nas condições e estilos de vida, cabendo parcela significativa da formulação e das responsabilidades ao denominado setor saúde.

Dentro da perspectiva médica, autores como Bausell (1998) julgam que, dada a grande abundância das atuais medidas de qualidade de vida, essas deveriam ser consideradas o ponto de partida para as políticas de atenção. Dechamp-Le Roux (1997) considera que a avaliação de qualidade de vida dá alma à tecnologização excessiva do setor. Porém, na medida que não leva em conta fatores sociais e econômicos, seu alcance passa a ser muito restrito, reproduzindo a lógica apenas biomédica.
Dentro do mesmo pensamento, Castiel (1995) comenta que um julgamento apenas econômico como o que domina o debate da qualidade de vida em saúde não pode ser ético. Tampouco seria ético desconsiderar o econômico no processo das escolhas, sobretudo em saúde, onde a tendência dos custos é sempre crescente.

Nos últimos dez anos, a utilização dos QALY vem sendo intensamente discutida, porque o problema dos custos tem que ser encarado, já que representam sacrifícios impostos a outros que não poderão ser tratados. Por outro lado, os que julgam ser imoral arbitrar o valor da vida de outros e preferem deixá-la ao azar da oferta de serviços assumem a pragmática idéia de que o primeiro a chegar é o primeiro a ser servido.

Maynard \& Bloom (1998) reiteram o consenso entre especialistas de que a escassez, em sua ubiqüidade, implica sempre a escolha de que qualquer prestação de serviço envolve a decisão de não oferecer um outro. Reconsiderando o problema das escolhas individuais de utilidade coletiva, duas hipóteses se colocam: a) todos os indivíduos do grupo social têm as mesmas preferências e assim se tenta determinar a utilidade atribuída a cada estado de saúde e b) as preferências diferem e a agregação escolhida deve ser claramente justificada, admitindo-se que alguns impõem suas preferências como ditadores invisíveis.

Partindo das idéias anteriores e corroborando a afirmativa de Rameix (1997), de que a medida da qualidade de vida no universo da saúde é irreversível, torna-se fundamental uma precaução para que sua utilidade, ao definir prioridades no racionamento de recursos, não seja confundida com a máquina de triturar oposições, com que Cevasco (1999) rotula uma das características eficazes do neoliberalismo. Por outro lado, torna-se necessário investir muito ainda no aprofundamento do conceito e da mediação de promoção da saúde para que signifique mais do que uma idéia de senso comum, programa ideológico, imagem-objetivo e possa nortear o sentido verdadeiramente positivo de qualidade de vida. 


\section{Referências bibliográficas}

Auquier P, Simeoni MC \& Mendizabal H 1997. Approches théoriques et méthodologiques de la qualité de vie liée à la santé. Revue Prevenir 33:77-86.

Auray J-P \& Duru G 1995. Qualité de Vie, pp. 83-128. In Moto (org.). Santé et Mutidisciplinarité. Choix et Décisions, Hermès, Paris.

Banco Mundial 1993. Relatório sobre o desenvolvimento mundial, 1993. Investindo em Saúde. Indicadores de desenvolvimento Mundial. Brasília, 347 pp.

Barata RB et al. 1997. Condições de vida e situação de saúde. Saúde Movimento, 4. Abrasco, Rio de Janeiro. Bausell RB 1998 Quality-of-life assessment in outcomes research. Evaluation \& The Health Professions 21(2):139-140.

Bley D \& Nernazza-Licht N 1997. La multiplicité usage du thèrme de qualité de vie. Revue Prevenir 33:713.

Bowling A 1994. La Medida de la Ssalud: Revisión de las Escalas de Medida de la Calidad de Vida. Masson, Barcelona.

Briggs A 1995. Handling uncertainty in the results of economic evaluation. Office of Health Economics (OHE) Briefing 32:1-12.

Breilh J, Granda E, Campaña A, Yépez J, Páez R, \& Costales P 1990. Deterioro de la Vida: Un Instrumento para Análisis de Prioridades Regionales en lo Social y la Salud. Corporación Editora Nacional, Quito.

Brunet-Jailly J 1997. L'évaluation économique des programmes de santé: est-elle éthique? Ruptures 4(1): 8-22.

Buss PM et al. 1998. Promoção da Saúde e Saúde pública. ENSP/Fiocruz, Rio de Janeiro. 178pp. (Mimeo).

Cardoso AL 1998. Indicadores sociais e políticas públicas: algumas notas críticas. Proposta 77:42-53.

Carrère MO, Duru G, Fervers B \& Spath HM 1997. Décision collective, évaluation économique et qualité de vie dans le secteur de la santé: entre pratique et théorie. Revue Prevenir 33:121-132.

Castellanos PL 1997. Epidemiologia, saúde pública, situação de saúde e condições de vida: considerações conceituais, pp. 31-76. In RB Barata (org.). Condições de Vida e Situação de Saúde. Saúde Movimento, 4. Abrasco, Rio de Janeiro.

Castiel LD 1995. Équité et Santé. Éditions ENSP, Rio de Janeiro.

Cevasco ME 1999. Máquina de moer oposições. Veredas 37:37-39.

Dasbach E \& Teutsch SM 1996. Cost-utility analysis, pp. 4-12. In AC Hadix, SM Teutsch, PA Shafer \& DO Dunet (orgs.). Prevention Effectiveness: a Guide to Decision Analysis and Economic Evaluation. Oxford University Press, Oxford.

Dechmp-Le Roux C 1997. Impact des technologies médicales sur la qualité de vie. Revue Prevenir 33:105111.

Dines A 1999. Balzac e o desenvolvimento. Jornal do Brasil. Primeiro Caderno, 22 de maio, p.11.

Durand D, Bley D \& Vernazza-Licht N 1997. Editorial. Revue Prevenir 33:3-6.

Engels F 1975. A Situação da Classe Trabalhadora na Inglaterra. Ed. Afrontamento, Porto.

Folha de S. Paulo 1999. Qualidade de vida em São Paulo. Indice Folha Especial, 5 de setembro, pp. 1-4.
Gianchello AL 1996. Health outcomes research in Hispaniccs/Latinos. Journal of Medical Systems 21(5): 235-254.

Gold MR et al.1996. Identifying and valuing outcomes, pp. 82-123. In AC Haddix, SM Teutsch, PA Shafer \& DO Dunet (orgs.). Prevention Effectiveness: a Guide to Decision Analysis and Economic Evaluation. Oxford University Press, Oxford.

Gold MR, Teutsch SM, McCoy KL \& Haddix AC 1997. Assessing outcomes in population health: moving in the field forward. American Journal of Preventive Medicine 13(1):3-5.

Green M 1995. Measuring quality of life, pp.108-124. In M.Green (org.). The economics of health care. Office of Health Economics (OHE). Cambridgeshire, Inglaterra.

Hartz ZMA \& Pouvoirville G 1998. Avaliação dos programas de saúde: a eficiência em questão. Ciência \& Saúde Coletiva - Revista da Abrasco 3(1):68-82.

Hinmam AR 1997. Quantitative policy analysis and public health policy: a macro and micro view. American Journal of Preventive Medicine 13(1):6-11.

Hubert A 1997. De la difficulté de définir une definition. Revue Prevenir 33:15-18.

Hyder AA, Rotlant G \& Morrow RM 1998. Measuring the burden of disease: healthy life-years. American Journal of Public Health 88(2):196-206.

Instituto de Pesquisa Econômica Aplicada, Instituto Brasileiro de Geografia e Estatística, Fundação João Pinheiro, Programa das Nações Unidas para o Desenvolvimento. Índice de Desenvolvimento Humano 1998. Indice de Desenvolvimento Humano (IDH) e Índice de Qualidade de Vida. IPEA, Brasília, 49 pp.

Lalonde M 1978. A New Perspective on the Health of Canadians: a Work Paper, 1974. Otawa. 76 pp.

Lebrun TH \& Sailly JC 1996. Qualité de vie et économie de la santé. Journal d'Économie Medicale no. hors série, outubro, $36 \mathrm{pp}$.

Léplege A \& Marciniack A 1997. Qualité de vie de ou santé subjective: problème conceptuels. Revue Prevenir 33:69-75.

Lima e Costa MF \& Sousa RP 1994. Qualidade de Vida: Compromisso Histórico da Epidemiologia. Coopmed/ Abrasco, Belo Horizonte.

Martin AJ \& Stockler M 1998. Quality of life assessment in health care research and practice. Evaluation \& Health Professions 21(2):141-156.

Matos O 1998. As formas modernas do atraso. Folha de S. Paulo, Primeiro Caderno, 27 de setembro, p. 3.

Maynard A \& Bloom K 1998. Our certaining fate: rationing in health care. Office of Health Economics (OHE), Londres.

Mckeown T 1982. El Papel de la Medicina: Sueño, Espejismo o Némesis? Siglo Veintiuno Ed., México.

Moatti JP 1996. Priorités de santé publique: les dangers d'une derive utilitariste. Actualité et Dossier en Santé Publique 17:38-40.

Morris J, Perez D \& Mcnoe B 1998. The use of quality of life data in clinical practice. Quality of Life Research 7:85-91.

Ministério da Saúde 1996. Promoção da Saúde: Cartas de Otawa, Adelaide, Sundsvalle e Santa Fé de Bogotá. MS/IEC, Brasília, 48 pp. 
Nuñez N 1994. Perfiles de mortalidad según condiciones de vida en Venezuela, pp.199-217. In MFL Lima e Costa \& RP de Sousa (orgs.). Qualidade de Vida: Compromisso Histórico da Epidemiologia. Coopmed/ Abrasco, Belo Horizonte.

Oleske DM 1995. Measurement issues in the use of epidemiologic data, pp. 21-32. In Epidemiology and the Delivery of Health Care Services. Pleem um Press, Nova York-Londres.

Paim JS 1997. Abordagens teórico-conceituais em estudos de condições de vida e saúde: notas para reflexão, pp. 7-32. In RB Barata (org.) Condições de Vida e Situação de Saúde. Saúde Movimento, 4, Abrasco, Rio de Janeiro.

Patrick DL \& Erickson P 1993. Health Policy, Quality of Life: Health Care Evaluation and Resource Allocation. Oxford University Press, Nova York.

Programa das Nações Unidas para o Desenvolvimento 1998. Informe sobre o desenvolvimento humano. PNUD, Brasília, 280 pp.

Rameix S 1997. Justifications et difficultés éthiques du concept de qualité de vie. Revue Prevenir 33:89-103.

Rosen G 1980. Da Polícia Médica à Medicina Social. Editora Graal, Rio de Janeiro.
Rufino Netto A 1994. Qualidade de vida: compromisso histórico da epidemiologia, pp.11-18. In MFL Lima e Costa \& RP Sousa (orgs.). Qualidade de Vida: Compromisso Histórico da Epidemiologia. Coopmed/ Abrasco, Belo Horizonte.

Schalock RL 1995. Outcome-Based Research. Pleem um Press, Londres.

Schlenk EA et al. 1998. Health related quality of life in chronic disorders: comparison across studies using the MS SF-36. Quality of Life Research 7:57-65.

Sieguel PZ, Moriarty DG \& Zack MM 1996. A new tool for measuring and monitoring quality of life in the United States. Journal d'Économie Médicale. No. hors série (octobre), $63 \mathrm{pp}$.

Sigerist H 1946. The social sciences in the medical school, pp. 35-72. In Sigerist, H. (org.). The University at the Crossroad. Henry Schumann Publisher, Nova York.

WHOQOL Group 1995. The World Health Organization Quality of Life Assessment (WHOQOL): position paper from the World Health Organization. Social Science and Medicine 41(10):1.403-1.409.

Witier PL 1997. La qualité de vie. Revue Prevenir 33:6162 